\title{
Kinetic Analysis of the Hydrolysis of Methyl Parathion using Citrate-stabilized 10 nm Gold Nanoparticles
}

\author{
Rafaela Nita, ${ }^{\mathrm{a}}$ Scott A. Trammell, ${ }^{\mathrm{b}}$ Gregory A. Ellis, ${ }^{\mathrm{c}}$ Martin H. Moore, ${ }^{\mathrm{b}}$ Carissa M. Soto, ${ }^{\mathrm{b}}$ Dag- \\ mar H. Leary, ${ }^{b}$ Jake Fontana, ${ }^{b}$ Somayeh F. Talebzadeh ${ }^{a}$ and D. Andrew Knight*a \\ ${ }^{a}$ Chemistry Department, Florida Institute of Technology, 150 West University Boulevard, Melbourne, Florida, 32901, USA. \\ ${ }^{b}$ Center for Bio/Molecular Science and Engineering, Naval Research Laboratory, 4555 Overlook Avenue, SW, Washington, DC \\ 20375, USA. \\ ${ }^{c}$ National Academy of Sciences, National Research Council, Postdoctoral Research Associate, Naval Research Laboratory, 4555 \\ Overlook Avenue, SW, Washington, DC 20375, USA.
}

Abstract "Ligand-free" citrate-stabilized $10 \mathrm{~nm}_{6}$ gold nanoparticles (AuNPs) promote the hydrolysis of the thiophosphate ester methyl parathion $(\mathrm{MeP})_{8}$ on the surface of gold as a function of $\mathrm{pH}$ and two, temperature values. At $50{ }^{\circ} \mathrm{C}$, the active surface ${ }_{\circ}$ gold atoms show catalytic turnover $\sim 4$ times after $8_{1}$ hours and little turnover of gold surface atoms at $25_{2}$ ${ }^{\circ} \mathrm{C}$ with only $40 \%$ of the total atoms being active ${ }_{63}$ From Michaelis-Menten analysis, $k_{\text {cat }}$ increases be tween $\mathrm{pH} 8$ and 9 and decreases above $\mathrm{pH} 9$. As global analysis of the spectral changes confirmed. the stoichiometric reaction at $25^{\circ} \mathrm{C}$ and the cata lytic reaction at $50{ }^{\circ} \mathrm{C}$ and mass spectrometry con $\triangleleft s$ firmed the identity of $p$-nitrophenolate $(P N P)$ prod ${ }^{69}$ uct. Additional decomposition pathways involving ${ }^{70}$ oxidation and hydrolysis independent of the forma ${ }^{71}$ tion of $P N P$ were also seen at $50{ }^{\circ} \mathrm{C}$ for both catalyzed and un-catalyzed reactions. This work represents the first kinetic analysis of ligand-free AuNP catalyzed hydrolysis of a thiophosphate ester.

\section{Introduction}

Methyl parathion (MeP, $O, O$-dimethyl- $O-p$ nitrophenyl phosphorothioate) is a broad spectrum organothiophosphate insecticide and hazardous pollutant. Both $\mathrm{MeP}$ and the analogous ethyl parathion continue to be used extensively (and often illegally in some countries) for the treatment of large-scale agricultural crops such as cotton, soybean, corn and wheat as well as other cultivated fruits and vegetables.(Tabassum et al., 2014) The accumulation of $\mathrm{MeP}$ and its degradation products in the environment is considered to be a significant widespread threat to regional ecologies. MeP also serves as a structural mimic for organophosphate nerve agents, and is often used as a model substrate for studies on the catalytic degradation of chemical ${ }^{6}$ warfare agents, which also act as acetylcholinesterase inhibitors (e.g. VX, soman, tabun).(Khanna, 2008) Thus, new methods for MeP removal from the environment, and studies on the mechanistic pathway(s) for MeP breakdown are of great interest to environmental scientists. The degradation of $\mathrm{MeP}$ can proceed through both oxi- dative and hydrolytic pathways under basic conditions, and can occur both photochemically and in the dark. At least 20 breakdown/rearrangement products have been identified using NMR or MS.(Seger and Maciel, 2005) Non-photochemical reaction products are reproduced in Figure 1.(Seger and Maciel, 2005) Hydrolysis can proceed through either loss of the methoxy or p-nitrophenoxy groups to give thiophosphate ions $\mathbf{1}$ and $\mathbf{2}$ (shown in the protonated form). $O, S$-isomerization of MeP gives $S$-Me and $S$-Ar species $\mathbf{3}$ and $\mathbf{4}$, and oxidation results in the oxon 5.

Fig. 1. Initial non-photochemical breakdown products of methyl parathion. Tautomeric forms of $\mathbf{1}$ and $\mathbf{2}$ are not shown.

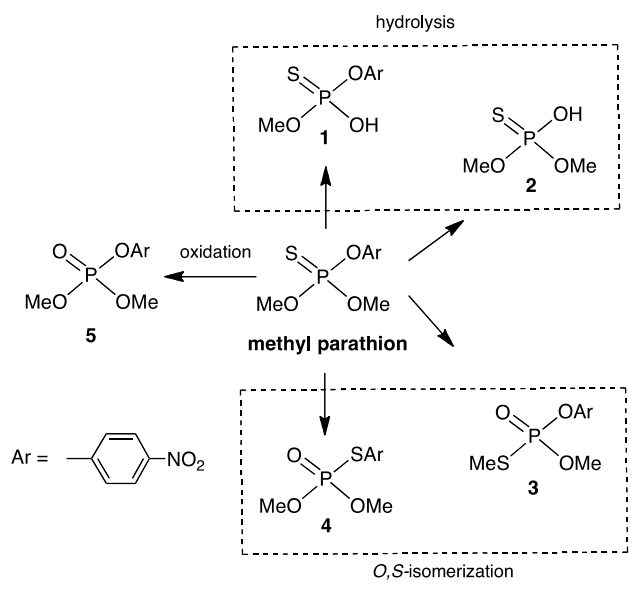

Ligand stabilized gold nanoparticles (AuNPs) are known to catalyze organic transformations under homogeneous conditions and commonly require either thiol, amine or phosphine ligands in which the ligand donor atom is covalently bound to the AuNP surface.(Zhang et al., 2011) CTAB stabilized AuNPs have been used for the detection of $\mathrm{MeP}$ at the ppm level by following its degradation products.(Barman et al., 2013a) Applications of "ligand-free" or anion-stabilized gold colloids in catalysis are rare. Barman et al reported the use of bio-fabricated AuNPs as a colorimetric sensor for 


\section{Results and discussion} dation of MeP using commercially available $10 \mathrm{~nm}_{5}$ AuNP under several conditions were monitored ${ }^{6}$ using the absorbance of the product $p^{\text {u }}$ nitrophenolate $(P N P)$ anion at $405 \mathrm{~nm}$ (Figure 2).48 Mass spectral analysis of the reaction confirmed the hydrolysis products $P N P$ and $\left[(\mathrm{MeO})_{2} \mathrm{P}(\mathrm{S})(\mathrm{OH})\right] \mathrm{in}^{150}$ addition to products due to oxidation and alternative hydrolytic pathways (Supporting Information). ${ }^{31} \mathrm{P}$ NMR analysis provided confirmation of product ${ }^{153}$ identities (Supporting Information). The reactions were performed in $20 \mathrm{mM}$ MOPS or CHES buffer with $5 \%$ methanol to aid solubilization of $\mathrm{MeP}^{16}$ which has a low solubility in water. At $25^{\circ} \mathrm{C}$, control reactions performed in the absence of AuNPs showed no formation of $P N P$, however at $50{ }^{\circ} \mathrm{C}$, a small degree of background hydrolysis was observed. The kinetic experiments confirm that AuNPs accelerate the degradation of MeP to $P N P^{162}$. At $25^{\circ} \mathrm{C}$ the data fits to a single exponential rise to ${ }^{163}$ a maximum value of $[P N P]=4.9 \mathrm{M}$ corresponding to $\sim 41 \%$ of the total number of surface gold atoms $(12 \mathrm{M})$. At $50^{\circ} \mathrm{C}$ the exponential rise levels off to a linear region and after 200 minutes, $[P N P]=21_{68}$ $M$ suggesting that the "active gold" surface atoms, have undergone turnover several times. From the linear region of $[P N P]$ vs. time, we used a $\mathrm{Micha}_{\bar{\Gamma} 11}$ elis-Menten formalism normalized to the active molar amount of surface gold atoms adjusting the $\mathrm{MeP}$ concentration for attachment to AuNP. The ${ }_{4}$ $10 \mathrm{~nm}$ AuNP core diameter was confirmed by TEM, (Supporting Information) and a Spherical Cluster ${ }_{6}$ Approximation (SCA)(Johnston, 2002) was used $_{s 7}$ from which we calculated a value of $3,936 \mathrm{Au}$ at ${ }_{\overline{118}}$ oms. We make the assumption that the spherical nanoparticles remain intact throughout the course
The reactions for the non-photochemical degra ${ }_{\overline{144}}^{143}$

of the kinetic analysis. Table 1 lists the resultant $\mathrm{K}_{\mathrm{M}}$ and $\mathrm{k}_{\text {cat }}$ at $50{ }^{\circ} \mathrm{C}$ for several $\mathrm{pH}$ values.
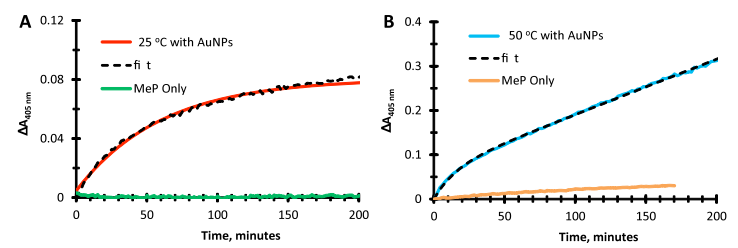

Fig. 2. The formation of nitrophenolate $(P N P, 405 \mathrm{~nm})$ from $\mathrm{MeP}([\mathrm{MeP}]=0.2 \mathrm{mM})$ under several conditions for the first 200 minutes. A. $\mathrm{pH} 8.3$ at $25^{\circ} \mathrm{C}$ : green $=\mathrm{MeP}$, red $=\mathrm{MeP}+$ citrate-stabilized $10 \mathrm{~nm}$ AuNPs, [AuNP surface atoms $]=12 \mathrm{M}$. The dashed line is a fit to a single exponential. B. $\mathrm{pH} 8.5$ at $50{ }^{\circ} \mathrm{C}$, : orange $=\mathrm{MeP}$, blue $=\mathrm{MeP}+$ citrate-stabilized $10 \mathrm{~nm}$ AuNPs The dotted line is a fit to a single exponential plus a linear function. $\left[\mathrm{AuNP}_{\text {surface atoms }}\right]=16 \mathrm{M}$. Table 1 .

Table 1. Kinetic analysis of the hydrolysis of MeP by AuNPs. ${ }^{\mathrm{a}} \mathrm{pH}$ values corrected for temperature at $50^{\circ} \mathrm{C}$.

Of note is the $\mathrm{pH}$ dependence of $\mathrm{k}_{\mathrm{cat}}$ which increases between $\mathrm{pH} 8$ and 9 , followed by a slow decrease (Figure 3). This suggests that $\mathrm{OH}^{-}$is playing an active role, however, at a $\mathrm{pH}$ value higher than 9, the AuNP surface exhibits decreased activity which we do not currently understand.

To obtain a better understanding of the nature of the spectral changes during the course of the reaction, we examined the changes between 260 to 800 $\mathrm{nm}$ showing both the forma-

\begin{tabular}{|r|c|c|c|}
\hline $\mathbf{p H}$ & $\boldsymbol{k}_{\text {cat}}, \mathbf{s}^{-1}$ & $\mathbf{K}^{\mathbf{M}}, \mathbf{M}$ & $\boldsymbol{k}_{\text {cat }} / \mathbf{K}_{\mathbf{M}}, \mathbf{M}^{-1} \mathbf{s}$ \\
\hline 7. & $2 \pm 1 \times 10^{-5}$ & - & - \\
\hline 8. & $1.8 \pm 0.2 \times 10$ & $2 \pm 1 \times 10^{-4}$ & - \\
\hline 8. & $4.1 \pm 0.3 \times 10$ & $1.0 \pm 0.2 \times 10$ & 4.0 \\
\hline 9. & $3.9 \pm 0.3 \times 10$ & $5.6 \pm 3.1 \times 10$ & 6.9 \\
\hline 9. & $2.6 \pm 0.3 \times 10$ & $4.2 \pm 1.6 \times 10$ & 6.9 \\
\hline
\end{tabular}
tion of $P N P$, and the changes in the MeP and AuNP spectra as the reaction progressed. We used the software ReactLab ${ }^{\mathrm{TM}}$ KINETICS which provides global analysis for fitting chemical reaction schemes and their parameters to spectroscopic data. In these experiments, we chose reaction conditions which allowed the recording of a measureable absorbance in the UV range and gave tractable concentrations of MeP. In addition, a pH 9 value was selected which exhibited considerable stability in regard to AuNP aggregation compared to lower $\mathrm{pH}$ values.

\footnotetext{
a Ligand-free citrate-stabilized $10 \mathrm{~nm}$ Au nanoparticles are referred to as AuNP throughout.
}

\footnotetext{
b http://www.liv.ac.uk/buffers/buffercalc.html
} 

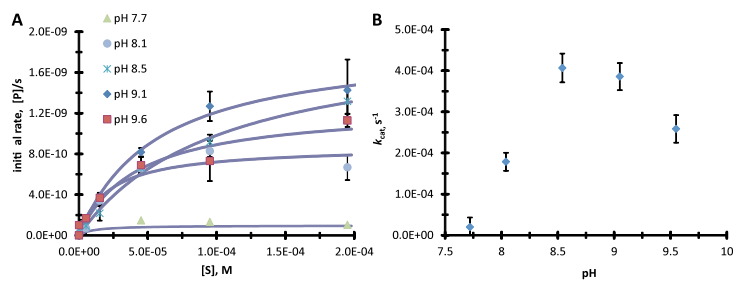

Fig. 3. A. The rate of $P N P$ formation vs. MeP concentration fitits to a Michaelis-Menten formalism at several $\mathrm{pH}$ values with $[\mathrm{AuNP}$ active surface atoms $]=4.9 \mathrm{M}$ at $50^{\circ} \mathrm{C}$. B. $k_{\text {cat }}$ vs. pH. Buffer $=20 \mathrm{mM}$ MOPS ( $\mathrm{pH} 7.7$ to 8.1) and CHES (pH 8.5 to 9.6) ${ }_{231}^{230}$ Assays were performed in triplicate.

At pH 8 in $20 \mathrm{mM}$ MOPS buffer, the addition ${ }^{233}$ $\mathrm{MeP}$ to $10 \mathrm{~nm}$ Au colloid results in an immediate $6^{24}$ $\mathrm{nm}$ red shift in the plasmon band which indicates an interaction of the thiophosphate substrate with the Au surface. After 8 hours, large $(>1 \mathrm{~m})$ irregu $^{{ }^{23}}$ lar aggregates were isolated following centrifuga ${ }_{239}^{238}$ tion.

Figure 4A shows the spectral characteristics of the MeP and AuNP reaction at $\mathrm{pH} 9$ and $50{ }^{\circ} \mathrm{C}_{2,21}^{24}$
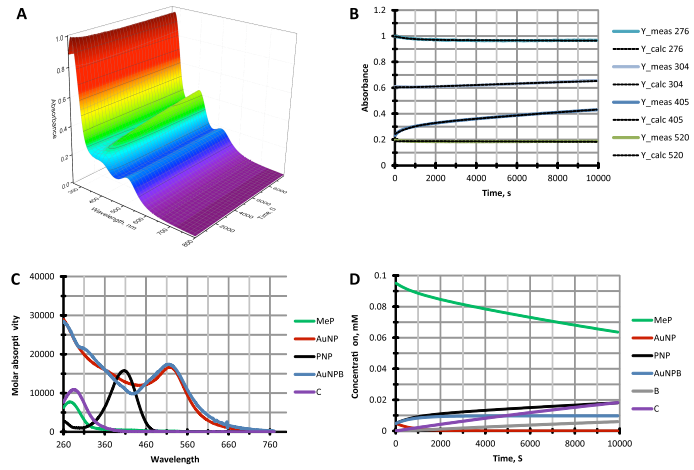

Fig. 4. A. A representative experiment showing the UV-Vis spectral changes for the reaction of MeP with AuNP over a 2.8 hour period $\left(1 \times 10^{-4} \mathrm{M} \mathrm{MeP}, 4.9 \times 10^{-6} \mathrm{M}\right.$ AuNP active surface atoms at $\mathrm{pH} 9\left(0.02 \mathrm{M}\right.$ CHES buffer) and $5 \% \mathrm{MeOH}$ at $50{ }^{\circ} \mathrm{C}$. B. The fitted plots of absorbance vs. time for several selected wavelengths. C. The UV-Vis spectra of the reactants, and products used in the analysis. AuNP and PNP were entered aso known spectra. B was entered as non-colored and C was entered as known spectra from the analysis of Figure S2. D. The concentration profiles calculated from fitted the data. In the modelling, $\mathrm{k}_{1}=13.9 \pm 0.07 \mathrm{M}^{-1} \mathrm{~s}^{-1}$ and $\mathrm{k}_{2}=0.85 \pm 0.001 \mathrm{M}^{-1} \mathrm{~s}^{-1}$ for equations 1 and 2 listed in the text. Background rates for equa264 tion $3\left(\mathrm{k}_{3}=3.2 \pm 0.02 \times 10^{-6} \mathrm{~s}^{-1}\right)$ and equation $4\left(\mathrm{k}_{4}=2.4 \pm 0.01_{65}\right.$ $\mathrm{x} 10^{-5} \mathrm{~s}^{-1}$ ) were used in the analysis from the control experiment without AuNPs (Supporting Information Figure S2).

The UV-Vis spectra change as a function of time reflecting the formation of the PNP product at ${ }^{9}$ $405 \mathrm{~nm}$ concomitant with changes in the $\mathrm{MeP}$ and ${ }^{\circ 0}$ AuNP spectra. Using the software ReactLab ${ }^{\mathrm{TM}^{\mathrm{T}}}$ KINETICS, we analysed the spectral changes using the model shown in eq. $1-4$. We propose this model based on the observations from wavelength ${ }^{24}$ data at $405 \mathrm{~nm}$ which suggests a catalytic step at 50 ${ }^{\circ} \mathrm{C}$. In the global model, we did not include more detailed elementary steps like on/off rates or equilibrium, hence the observed $2^{\text {nd }}$ order rate constants from the analysis are more complex.

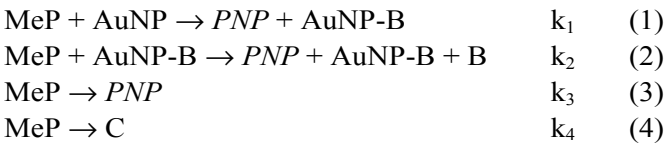

Equation 1 represents a 2 nd order reaction in which MeP reacts with a surface site on the AuNP to form the product $P N P$ and a new surface site AuNP-B. The reaction in eq. 2 includes the catalytic step in which the surface product on the gold, AuNP-B, is displaced by an incoming MeP molecule and then regenerated after hydrolysis. We used the number of active surface atoms as the molar concentration of the AuNP. Equations 3 and 4 correspond to the background decomposition pathways measured without AuNP (see Supporting Information). Figure 5B, shows the quality of the fit of the model to the data at several selected wavelengths by comparing the experimental data to the calculated results. Figure 5C shows the UV-Vis spectra of the reactants and products used in the analysis while Figure 5D shows the concentration profile of the reactants and products as the reaction progresses. In the supporting information, the global analysis at $25^{\circ} \mathrm{C}$ with AuNP shows the fit of the data with and without a catalytic step (eq 1) and a slow background hydrolysis rate (eq 3 ). The spectral analysis of MeP hydrolysis without AuNP at 25 ${ }^{\circ} \mathrm{C}$ and $50{ }^{\circ} \mathrm{C}$ is shown in Supporting Information. At $50{ }^{\circ} \mathrm{C}$, we see an additional product, (C) in the UV spectra with a $\lambda_{\max }$ at $285 \mathrm{~nm}$ in addition to the decrease of MeP with growth of the PNP peak for both un-catalyzed and catalyzed reactions at $50{ }^{\circ} \mathrm{C}$. The product $\mathrm{C}$ is not seen at $25^{\circ} \mathrm{C}$ for either the uncatalyzed or catalyzed reactions (Supporting Information). Likely candidates include the hydrolysis product 1 or the oxidation product 5 shown in Figure 1. Qualitative mass spectral analysis of dichloromethane extractions of acidified reaction mixtures (20 mM CHES buffer, 5\% methanol) confirmed the presence of the hydrolysis product $P N P$, the starting material MeP, and putatively the hydrolysis product $\mathbf{1}$, the oxidation product $\mathbf{5}$, and less confidently the $O, S$-isomerization products 3 or $\mathbf{4}$ Supporting Information). Mass spectral analysis of direct reaction mixtures (20 mM CHES buffer, 5\% methanol) also showed the reduction of starting material MeP and the accumulation of the hydrolysis product $P N P$ over time. Overall concentrations 

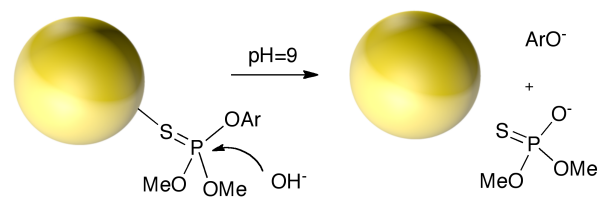

Fig. 5. One possible mechanism for activation of methyl parathion by $10 \mathrm{~nm}$ Au towards nucleophilic attack by the hydroxide ion releasing $p$-nitrophenolate (here shown as $\mathrm{ArO}^{-}$).

\section{Conclusions}

We have described a kinetic analysis of the catalytic degradation of the organothiophosphate pesticide methyl parathion in aqueous solution mediated by commercially available ligand-free citrate stabilized $10 \mathrm{~nm}$ gold nanoparticles. Temperature and $\mathrm{pH}$ dependencies are also described. Mass spectral data and ${ }^{31} \mathrm{P}$ NMR analysis indicate that the catalytic reaction does not follow a simple reaction but a more complex pattern in which alternative hydrolytic and oxidative pathways are followed. To the best of our knowledge this work represents the first kinetic analysis of ligand-free AuNP catalyzed hydrolysis of a thiophosphate ester. Experiments are underway to determine the details of the mechanism for the catalytic hydrolysis/oxidation.

Acknowledgements This work received support from the Defense Threat Reduction Agency-Joint Science and Technology Office for Chemical and Biological Defense (MIPR \#B102405M, B112542M and HDTRA136555. D.A.K is grateful to the American Society of Engineering Education and Office of Naval Research for a Distinguished Faculty Fellowship.

\section{Appendix A. Supplementary Material}

Supplementary data asssociated with this article can be found, in the online version, at:

\section{References}

Barman, G., Maiti, S., K. Laha, J., 2013a. Trichloroacetic acid assisted synthesis of gold nanoparticles and its application in detection and estimation of pesticide. J. Anal. Sci. Tech. 4, 1-7.

Barman, G., Maiti, S., Laha, J.K., 2013b. Bio-fabrication of gold nanoparticles using aqueous extract of red tomato and its use as a colorimetric sensor. Nanoscale Res. Lett. 8, 1-9.

Johnston, R.L., 2002. The Spherical Cluster Approximation. in: Betts, D.S. (Ed.). Atomic and Molecular Clusters. Taylor and Francis, New York, NY.

Khanna, V.K., 2008. Nanoparticle-based sensors. Def. Sci. J. 58, 608616.

Seger, M.R., Maciel, G.E., 2005. NMR investigation of the behavior of an organothiophosphate pesticide, methyl parathion, sorbed on clays. Environ. Sci. Technol. 40, 552-558.

Suramanee, P., Poompradub, S., Rojanathanes, R., Thamyongkit, P., 2011. Effects of Reaction Parameters in Catalysis of Glycerol Oxidation by Citrate-Stabilized Gold Nanoparticles. Catal. Lett. 141, 1677-1684.

Tabassum, N., Rafique, U., Balkhair, K.S., Ashraf, M.A., 2014. Chemodynamics of Methyl Parathion and Ethyl Parathion: Adsorption Models for Sustainable Agriculture. Bio. Med. Res. Int. 2014, 1-8.

Wang, S., Chen, W., Liu, A.-L., Hong, L., Deng, H.-H., Lin, X.-H., 2012. Comparison of the Peroxidase-Like Activity of Unmodified, Amino-Modified, and Citrate-Capped Gold Nanoparticles. Chem. Phys. Chem. 13, 1199-1204.

Zhang, Y., Cui, X., Shi, F., Deng, Y., 2011. Nano-Gold Catalysis in Fine Chemical Synthesis. Chem. Rev. 112, 2467-2505.

Zimmermann, L.M., Almerindo, G.I., Mora, J.R., Bechtold, I.H., Fiedler, H.D., Nome, F., 2013. Degradation of Methyl Paraoxon in the Presence of Mg2+-A13+ Mixed Oxides. J. Phys. Chem., C 117, 26097-26105. 